\title{
Texture Segmentation Based on Multifractal Dimension
}

\author{
AzmiTawfik Alrawi ${ }^{1}$, Ali makki Sagheer ${ }^{2}$ and Dheyaa Ahmed Ibrahim ${ }^{3}$ \\ ${ }^{1}$ Department of physics, University of Anbar, Ramadi, Iraq \\ Dr_azm_alrwi@yahoo.com \\ ${ }^{2}$ Department of Information System, University of Anbar,Ramadi,Iraq \\ ali_makki_sagheer@yahoo.com \\ ${ }^{3}$ Department of Computer Science, University of Anbar, Ramadi, Iraq \\ dhaia_1988@yahoo.com
}

\begin{abstract}
Texture segmentation can be considered the most important problem, since human can distinguish different textures quit easily, but the automatic segmentation is quit complex and it is still an open problem for research. In this paper focus on implement novel supervised algorithm for multitexture segmentation and this algorithm based on blocking procedure where each image divide into block $(16 \times 16$ pixels $)$ and extract vector feature for each block to classification these block based on these feature. These feature extract using Box Counting Method (BCM). BCM generate single feature for each block and this feature not enough to characterize each block, therefore, must be implement algorithm provide more than one slide for the image based on new method produce multithresolding, after this use BCM to generate single feature for each slide.
\end{abstract}

\section{KEYWORDS}

Texture Segmentation; MultifractalDiension; Fractal Dimension; Box Counting

\section{INTRODUCTION}

Texture segmentation is a subject that has received significant attention in recent years. In texture segmentation, the goal is to divide an image into coherent regions using texture information. There are two types of texture segmentation supervised and unsupervised. In supervised texture segmentation, the system has models of textures to be encountered in the images to be segmented. Unsupervised texture segmentation, on the other hand, aims at dividing an image into regions of similar texture without a priori information about the different textures. Image segmentation, even though an ill problem has several practical applications and texture has proven to be a useful cue in segmentation [1].

Texture defect detection is a sub problem of texture segmentation, and it is commonly encountered in visual inspection [2]. Texture segmentation is a difficult problem because one usually does not have a prior knowledge about what types of textures exist in an image, how

DOI : $10.5121 /$ ijsc.2012.3101 
International Journal on Soft Computing ( IJSC ) Vol.3, No.1, February 2012

many different textures there are? And what textures exist in each region in the image? In fact, one does not need to know which specific textures exist in the image in order to do texture segmentation. What is needed to know is to tell that? There are two different textures in some adjacent regions of the image [3]. The two general approaches to perform texture segmentation are analogous to the image segmentation methods: region-based approaches or boundary-based

approaches. Strictly speaking, the boundary based methods result in segmentation only if all the detected boundaries form closed curves.

Texture analysis is one of the most important techniques used in the analysis and interpretation of images, consisting of repetition or quasi repetition of some fundamental image elements there are three primary issues in texture analysis, such as texture classification, texture segmentation and shape recovery from texture. In texture classification, the problem is identifying the given texture region from a given set of texture classes. As opposed to texture classification, in which the class label of a single homogeneous region is determined using distinguishing features derived from the region, texture segmentation is concerned with automatically determining the boundaries between various textured regions in an image [4].

\section{Previous Works}

T.Kasparis, 2001: This paper describes a new approach to the segmentation of textured gray-scale images based on image pre-filtering and fractal features. Traditionally, filter bank decomposition methods consider the energy in each band as the textural feature, a parameter that is highly dependent on image intensity. In this paper, we use fractal-based features which depend more on textural characteristics and not intensity information. To reduce the total number of features used in the segmentation, the significance of each feature is examined using a test similar to the F-test, and less significant features are not used in the clustering process. The commonly used K-means algorithm is extended to an iterative $\mathrm{K}$-means by using a variable window size that preserves boundary details. The number of clusters is estimated using an improved hierarchical approach that ignores information extracted around region boundaries [5].

DimitriosCharalampidis, 2002: In this paper, introduced a rotational invariant feature set for texture segmentation and classification, based on an extension of fractal dimension (FD) features. The FD extracts roughness information from images considering all available scales at once. In this work, a single scale is considered at a time so that textures with scale-dependent properties are satisfactorily characterized. Single-scale features are combined with multiple-scale features for a more complete textural representation. Wavelets are employed for the computation of single- and multiple-scale roughness features because of their ability to extract information at different resolutions. Features are extracted in multiple directions using directional wavelets, and the feature vector is finally transformed to a rotational invariant feature vector that retains the texture directional information. An iterative means scheme is used for segmentation, and a simplified form of a Bayesian classifier is used for classification. The use of the roughness feature set results in high-quality segmentation performance. Furthermore, it is shown that the roughness feature set exhibits a higher classification rate than other feature vectors presented in this work. The feature set retains the important properties of FD-based features, namely insensitivity to absolute illumination and contrast [6].

Hsu Taoi, 2009: An efficient algorithm for segmenting a textured image into different regions is developed based on a set of fractal dimension estimated from calculated variorum. An edge 
International Journal on Soft Computing ( IJSC ) Vol.3, No.1, February 2012

detector is employed to detect texture boundary based on the multi-resolution framework including gradient pyramid construction followed by the reliable information obtained in the rough resolution as mask to constrain the calculation region in the rest resolution. Combined with mask and gradient in each resolution is propagating down to the finest resolution giving a more accurate boundaries estimation. The utility of the proposed method is demonstrated on a number of synthesis and natural textures [7].

\section{The Proposed System}

The proposed segmentation system operates on texture images which are based on blocking concept. Blocking concept involves processing a texture images as a sections called blocks. These blocks have the same size across the texture image. Every pixel within each block was assigned the same texture values.

The proposed system segments the complicated texture surface based on true shape texture. The original texture image consists of groups of many texture types such as Wood, Stone and Grass. There are many techniques proposes for multi texture segmentation such as: statistical and structural coefficients. Such methods include first order statistics such as the variance or energy of each color band, second order statistics based on the co-occurrence matrices of coefficients, and random field models.

In this work, feature extraction is employed to use the Fractal Dimension FD instead of other method because the FD is insensitive to differences in the local intensity of the texture image. Ideally, the FD is also insensitive to image zooming but in practice this is only valid to a certain extent. The FD of single feature is not sufficient for texture analysis and characterization, therefore, it must be compute multifractal for texture analysis. The FD estimation using Box Counting Method (BCM) which is based on binarization method. The binarization method is achieved using multithresholding algorithm. The multithresholdingalgorithm can generate more than one slide for each texture image. The multifractal dimension idea is accomplished by apply single FD on each slide which is resulted from multithresholding algorithm. The multifractal dimension obtains feature vector $X=\{x 1, x 2, \ldots, x n\}$ for all pixel of texture image.

An image has been transformed bymultifractal dimension into more than one feature images and a pixel at any position has associated with a feature vector $X=\{x 1, x 2, \ldots, x n\}$, where there are $n$ feature images equal to number of slide. Feature vector is used to assign one texture to the pixel under examination given its $\mathrm{n}$ dimensional feature vector.

The proposed segmentation system is trained supervisedlly by the exporter. The human visual system of the exporter has a knowledge about the texture nature within the textures groups image. These system uses the knowledge which are detected to classify the textures pixels.

The general block diagram of the proposed segmentation system is illustrated below in Figure (1). It represents the general scheme of the segmentation process step by step. 


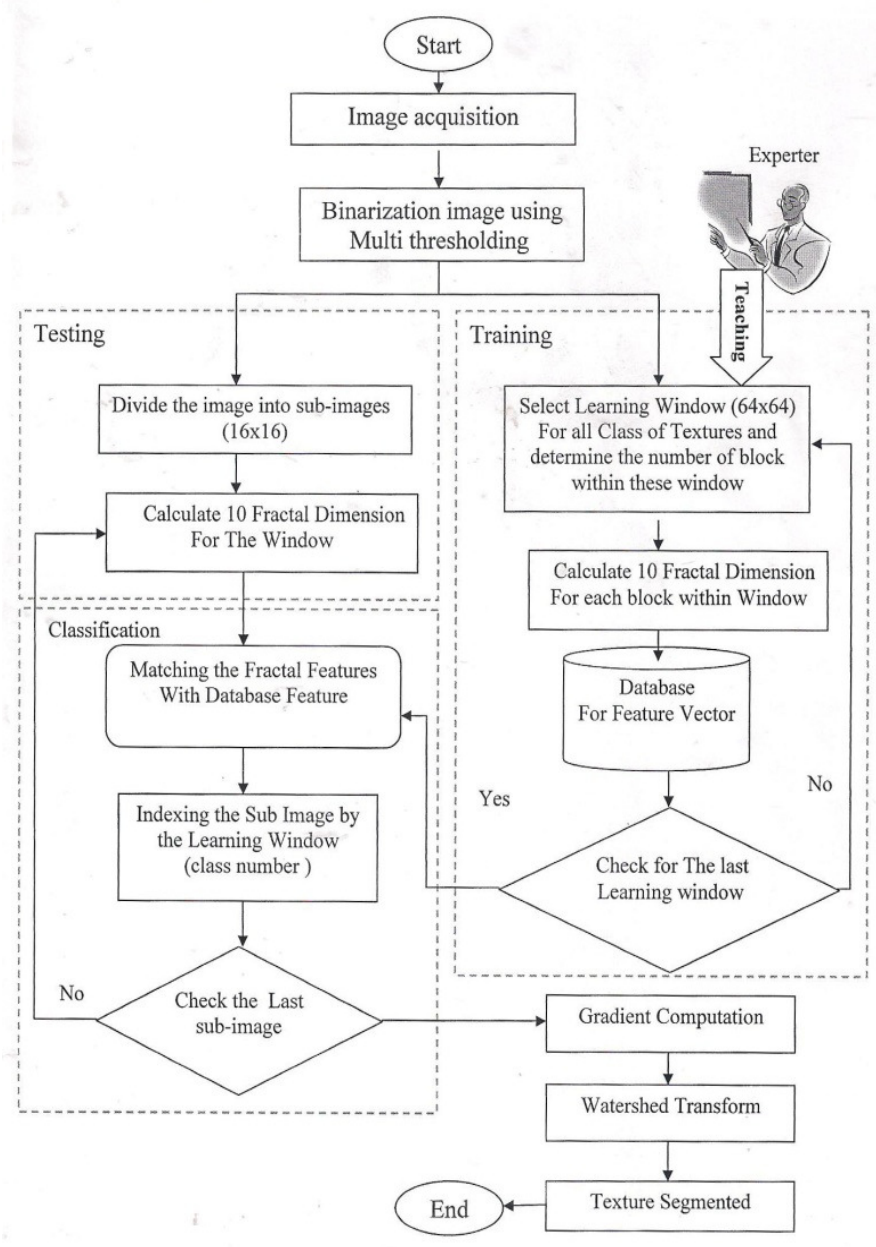

Figure (1) Proposed Technique Diagram for Texture Segmentation

\section{a. Image Acquisition}

The first and arguably most important stage is that of image acquisition. The application of suitable physical constraints to the observed scene may be used to significantly reduce the complexity of subsequent stage. Careful structuring of the lighting arrangement and camera position may be used to enhance the particular features of interest

\section{b. Binarization by Multithresholding}

After Image acquisition conversion of the color image to gray image. There are two methods to calculate threshold (histogram and traditional method ). First, Histogram shows the number of pixels for each gray value within the range of the image gray scale. If the minimum value of the image is 0 and the maximum value of the image is 255, then the histogram of the image shows the number of pixels for each gray value ranging between 0 and 255. Peaks in the histogram represent more common gray values within the image that usually consists of nearly uniform regions. Valleys in the histogram represent less common values. Empty regions within the 
International Journal on Soft Computing ( IJSC ) Vol.3, No.1, February 2012

histogram indicate that no pixels within the image contain gray values belong to these empty regions. The conversion from gray image to binary image could be done by using thresholding method. In threshold method, a threshold value should be defined. After compute the histogram the maximum and minimum value (i.e., low gray and high gray regions) indicate. This process differs from one method to other, for example in the traditional method the minimum and maximum existing values can be determined directly from the component itself while in the histogram thresholding method the minimum and maximum values are estimated as lower and upper bounds of the component values at which the sum of the histogram elements beyond these bounds is less than a specific predefined value. To calculate the set of uniformly distributed threshold values; a calculation of the intensity interval between the proposed threshold values. Algorithm 1 shows the steps binarization by multithresholding image using histogram method.

Algorithm (4.1):Thresholding a gray scale image using traditional thresholding method.

Input: Color image with height (H) \& width (W), No. of thresholds (N).

Output: (N) Intensity Thresholds (Tr).

\section{Begin}

Step 1: Finding the Min. \& Max. values.

Step 2: Calculate the intensity interval $(S)$ between the intensity thresholds.

$$
\mathrm{S}=\frac{\text { Max }- \text { Min }}{N+1}
$$

Step 3:Calculate Thresholds

$$
\text { For } I=1 \text { to } N
$$

$\operatorname{Tr}(I)=\operatorname{Min}+S * I$

Step 4:Print (N) thresholds for each color channel \& gray.

\section{End}

\section{c. Feature Extraction based on Fractal Dimension}

Instead of using the raw data, selected measurements called features extracted from the raw data are used as the basis for classification. Features should be designed to be invariant or less sensitive with respect to commonly encountered variations and distortions, whilst containing less redundancies. The criterion of feature extraction can be sensibly based on either:

The importance of the features in characterizing the patterns.

The contribution of the features to the performance of recognition.

The FD as a single feature is not sufficient for texture analysis and characterization. The idea of using more than one FD feature has already been introduced previously. The extraction of a set of features produces a feature vector i.e. a feature vector $\mathrm{X}$ will take the form: 
International Journal on Soft Computing ( IJSC ) Vol.3, No.1, February 2012

$$
\mathrm{X}=\left[\begin{array}{c}
\mathrm{FD}_{1} \\
\mathrm{FD}_{2} \\
\mathrm{FD}_{3} \\
\vdots \\
\mathrm{FD}_{\mathrm{n}}
\end{array}\right]
$$

Feature extrication implementation using Box Counting Method (BCM) for all slide generated from multithresholding algorithm. Also, must be allustratingthat the FD values computes over the block. In order to calculate the box-counting dimension, any structure should be located on square grid with the mesh dimension s, and afterwards, all meshes that contain even the smallest part of the structure are summed up. In this way, the number of filled meshes $\mathrm{N}$ is calculated, which is related to their dimension, therefore is registered as $\mathrm{N}(\mathrm{s})$. Next, each mesh divides into four equal ones and repeat the operation. In practice, the algorithm can be continued until which restricted by the device resolution.

In literature, for calculation of box dimension it does not matter if the number of boxes is counted for the entire block. In this work need to calculate the number of boxes A(s) for entire block to solve overlapping problem related with calculate the FD for these blocks. The fractal dimension is obtained using the following equation:

$$
\mathrm{H}=\frac{\sum_{\mathrm{S}=\mathrm{k}}^{2} \log (\mathrm{A}(\mathrm{s})) * \log (\mathrm{N}(\mathrm{s}))}{\sum_{\mathrm{s}=0}^{\mathrm{k}-2} \log (\mathrm{A}(\mathrm{S}))^{2}}
$$

$$
\mathrm{FD}=2-\mathrm{H}
$$

Where $\mathrm{H}$ is slop, $\mathrm{A}(\mathrm{s})$ is total number of boxes to all block, $\mathrm{N}(\mathrm{s})$ is number of box cover the structure, $\mathrm{k}$ is highest box size and $\mathrm{s}$ is box size. The FD of a curve can be ranging between 1 (which is equal to its topological dimension) and 2 (which is equal to the topological dimension of the plane that it can occupy).

\section{d. Learning Phase}

The supervised learning procedure is the most important part of the system for automatic classification mode. This phase is implemented by select window of size $\mathrm{LH} \times \mathrm{LW}$ by expert for each texture in image. The size of the window is important. As the window size gets larger, more global features are detected. This suggests that the choice of window size could possibly be tied to the contents of the image. The images with larger texture tokens would require larger window sizes whereas finer textures would require smaller windows. It is also possible to regard the window size as a space parameter and use a multi-scale filtering approach. The proposed technique has done experiments in both aspects. After this these window divided randomly into small blocks of size $\mathrm{M} \times \mathrm{M}$ to increase the amount of data for each texture class which increase the discrimination between classes in image, where $M$ must be greater than 0 and less than width and height of the selected window of size LH $\times$ LW. Start pixel for block can be detected using equation (3) and (4). For each one of this block computes N FD equal to the number of thresholding.

$$
\begin{gathered}
\mathrm{XS}=(\mathrm{LH}-\mathrm{M}) * \text { rand } \\
\mathrm{YS}=(\mathrm{LW}-\mathrm{M}) * \text { rand }
\end{gathered}
$$


International Journal on Soft Computing ( IJSC ) Vol.3, No.1, February 2012

Where XS , YS are the index for start pixel to blocks and rand randomly number between $(0,1)$. After this compute the feature vector for each block and use these feature for compute the mean and standard deviation for the selected window. As shone previously in this chapter, the proposed technique generates more than one slide of the image depending on the multithresholding algorithm, for each slide is calculated by fractal dimension. Therefore, number of mean and standard deviation (STD) equal number of thresholding for all selected window.ListenRead phonetically

The Mean is implemented by using the following equation.

$\operatorname{Mean}(\mathrm{T})=\frac{\sum_{\mathrm{i}=0}^{\mathrm{SW}} \mathrm{FD}(\mathrm{i})}{\mathrm{SW}}$

The STD is implemented by using the following equation

$\operatorname{STD}(T) \sqrt{\frac{\sum_{\mathrm{i}=1}^{\mathrm{SW}}(\mathrm{FD}(\mathrm{i})-\mathrm{mean}(\mathrm{T}))^{2}}{\mathrm{SW}-1}}$

Where $\mathrm{T}$ is the thresholding, and SW is the number of block within the selected window.

\section{e. Testing Blocks Phase}

Multi texture images of size $\mathrm{N} \times \mathrm{N}$ are considered. The analysis is carried out by considering subimages (i.e., block) of size $\mathrm{M} \times \mathrm{M}$. Each $\mathrm{M} \times \mathrm{M}$ sub-image, taken from top left corner of the original image and computes multifractal dimension for each block. After this the matrix has $\mathrm{N} \times \mathrm{M}$, where $\mathrm{N}$ is the number of feature elements in each vector (equal to the number of thresholding) and $\mathrm{M}$ is the total data size (the total number of blocks in the input image).

\section{f. Block-Based Texture Classification phase}

The most important stage of the classification process is the feature extraction stage, at which time the sample block is transformed into a much lower dimensionality feature vector. The vectors from all of the training block are then input to the classification system for training. Again, many different classifiers have been used, although some of them perform slightly better than others, generally the choice of classifier has the least effect on the overall performance of the system. Therefore, speed of training, ease of implementation, and suitability to a given task are more important factors in the choice of a classifier than is its raw performance.

In these phase, the mean and standard deviation for feature are extracted from the learning sample using the proposed feature extraction algorithm, and then compared the number of features which extracted from each testing block with the corresponding mean and standard deviation of all the texture classes k stored using distance equation (7). show Figure (2) illustrates these phase.

$\operatorname{Dist}(\mathrm{K})=\sum_{\mathrm{i}=0}^{\mathrm{T}} \frac{\operatorname{mean}(\mathrm{i})-\mathrm{FD}(\mathrm{i})}{\operatorname{STD}(\mathrm{i})}$ 
Where $\mathrm{T}$ is the number of thresholding, mean and STD is the mean and stander deviation for training sample and FD is fractal dimension for test block. The identification of block with the same class $\mathrm{k}$ in database is represented by selecting the smallest value of Dist(k).

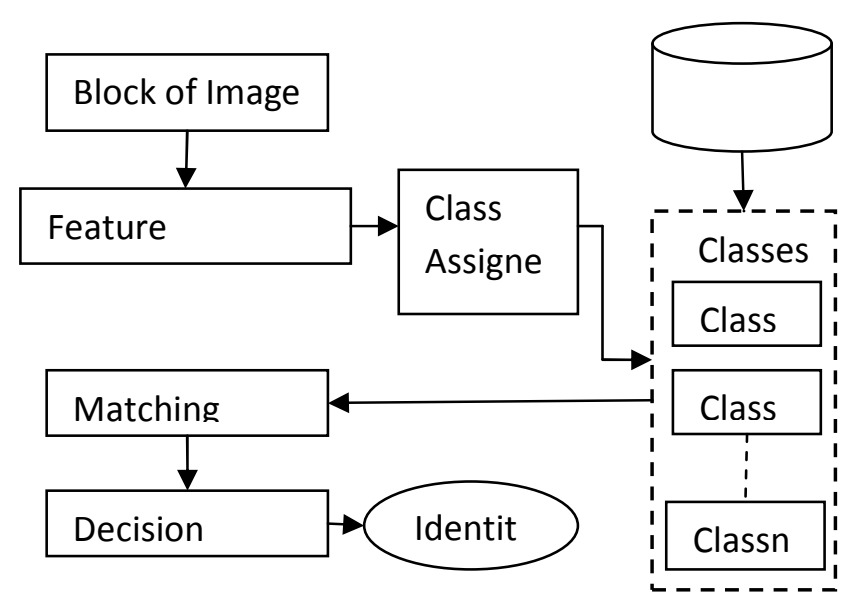

Figure (2) Block-Based Texture Classification phase

\section{EXPERIMENTAL RESULTS}

The segmentation technique discussed in the previous section is applied on six different texture. These images are a BMP 24 bit/pixels (bit depth), the size of each test image is $256 \times 256$ pixels. These images are consisting of two, three, four, and five textures. These textures extracted from various natural and surface texture images takes from world database. The collection of textures exhibits well defined variations to a given reference in terms of illumination, rotation and spatial resolution. There are some considerations related with multitexture design as follows:

The multitextur image consists of different paris of circles, rectangles and squares to evaluate its edges on the system performance properly. The size of textures within each image is configured randomly and irregularly measure that effect on results.

The multitexture image consist of more than two textures to scale the quality of segmentation. The texture type is natural or artificial.To measure the accuracy of the proposed segmentation method , there are supervised evaluation method. This measure takes into account the information that brings the ideal segmentation or "ground truth". Let $S=\{S 1, S 2, S 3, \ldots ., S m\}$ be the obtained segmentation and let $\mathrm{I}=\{\mathrm{I} 1, \mathrm{I} 2, \mathrm{I} 3, \ldots . ., \mathrm{Im}\}$ be the ideal segmentation of the same image. The problem of finding which Si corresponds to each Ii can be achieved by solving a maximum cost pairing problem between the regions on $\mathrm{S}$ and the regions on I. Each region can be obtained a coincidence index given by equation:

$$
\mathrm{Ci}=\frac{\left|\mathrm{S}_{\mathrm{i}} \cap \mathrm{I}_{\mathrm{i}}\right|}{\left|\mathrm{S}_{\mathrm{i}} \cup \mathrm{I}_{\mathrm{i}}\right|} \ldots[8](8)
$$

Where Si and Ii are supposed to represent the same region on the original image. The impact of the region on the global segmentation can be measured by computing: 


$$
\mathrm{Di}=\frac{\left|\mathrm{S}_{\mathrm{i}} \cap \mathrm{I}_{\mathrm{i}}\right|}{\mathrm{N}} \ldots[8](9)
$$

Where $\mathrm{N}$ stands for the total number of pixels of the image. We define the Coincidence Measure (CM) between $\mathrm{S}$ and I as:

$$
\mathrm{CM}=\sum_{\mathrm{i}=1}^{\mathrm{n}} \mathrm{D}_{\mathrm{i}} \mathrm{C}_{\mathrm{i}} \ldots[8](10)
$$

It is not difficult to see that $0 \leq \mathrm{CM} \leq 1$ and that for $\mathrm{CM}=1$ there is a perfect coincidence between I and S. Figure 3 show the result of image with $\mathrm{CM}$ for each image.

\section{CONCLUSION}

A novel approach based on fractal dimension as a feature for supervised texture segmentation is presented in this paper. These approach based on dividing the image into blocks. The fractal dimension values of the blocks are computed using box counting based on binarization using multithreshoolding to generate vector features. The size of the selected training window plays an important role in the supervised segmentation processes, the most suitable size, found in this research, was $(64 \times 64)$ pixels which gave higher segmentation accuracy when using the box counting method. The test results indicate that the FD feature is useful to assist the multitexture segmentation tasks. The performance of the system increases when the number of features increases but the performance of this system decreases when the number of features bigger than 10. The reason that the interval between the number of thresholding is fewer. The test results indicate that block size is useful to assist the multitexture segmentation tasks. The attained block size led to maximum quality segmentation rate ( with $8 \times 8$ block, with $16 \times 16$ block, with $32 \times 32$ block) and the best result with $(16 \times 16$ block).

\section{REFERENCE}

[1] Vincent Levesque, Texture Segmentation Using Gabor Filters, Msc Thesis, Center For Intelligent Machines, McGill University, 2009.

[2] TimoAhonen, Face And Texture Image Analysis With Quantized Filter Response Statistics, Dissertation, ActaUnivOul C 330, 89 p., 2009.

[3] Kamal H. Sager, Fractal Based Classification for Color Textural Images, PhD Thesis, Department of Astronomy and Space, College of Science, University of Baghdad, 2006.

[4] MihranTuceryan and Anil K.Jain, Texture Analysis, The Handbook of Pattern Recognition and Computer Vision, pp.207-248,1998.

[5] T.Kasparis, Segmentation of textured images based on fractals and image filtering, Pattern Recognition 34, 1963-1973,2001.

[6] DimitriosCharalampidis, Wavelet-Based Rotational Invariant Roughness Features for Texture Classification and Segmentation, IEEE TRANSACTIONS ON IMAGE PROCESSING, VOL. 11, NO. 8, AUGUST 2002.

[7] Hsu Taoi, Multi-resolution texture segmentation using fractal dimension, Journal of Communication and Computer, Volume 6, No.11. 2009. 
International Journal on Soft Computing ( IJSC ) Vol.3, No.1, February 2012

[8] Sandro Vega Pons, José Luis Gil Rodríguez and et al., Active Contour Algorithm for Texture Segmentation Using a Texture Feature Set, Advanced Technology Application Center, 2009
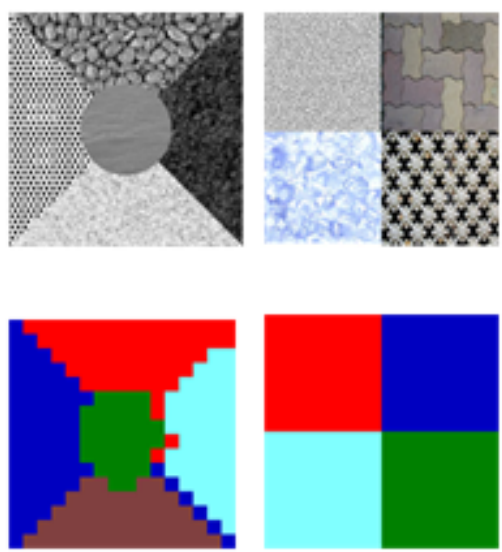

$\mathrm{CM}=0.83$

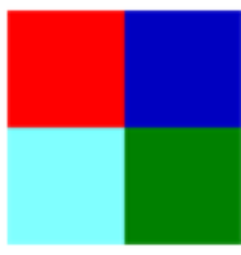

$\mathrm{CM}=0.98$
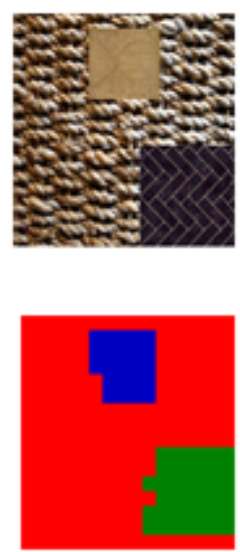

$\mathrm{CM}=0.91$
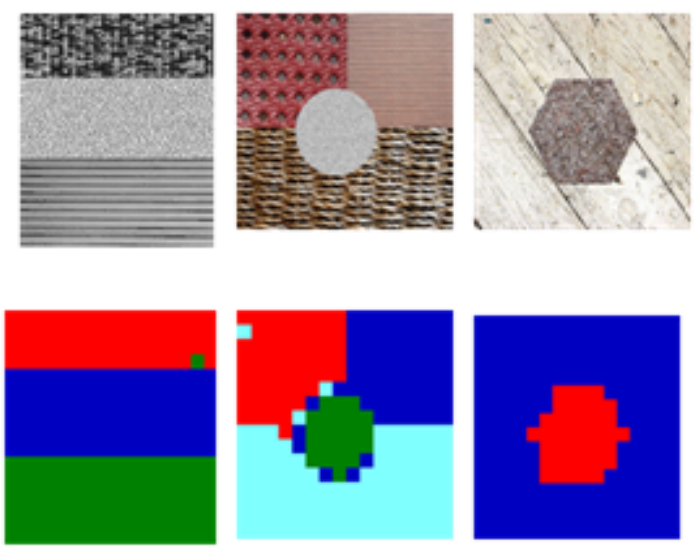

$\mathrm{CM}=0.94$

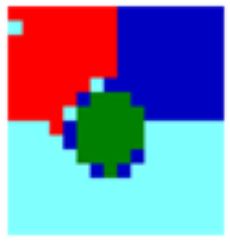

$\mathrm{CM}=0.86$

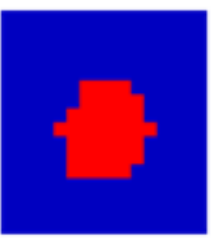

$\mathrm{CM}=0.91$

Figure (3). Illustrate the result of the proposed system

\section{Authors}

${ }^{\mathbf{1}}$ Azmi TawfikHussain was born in Anbar - 1961. He got on B.Sc of physical in salhdee university 1983 - Iraq and M.Sc. in theoretical physics in al yarmook university 1992 - Jordan and Ph.D. in Image Processing from in al-mustinsary university - Iraq, (2001). He interesting in the follow filed (fractal geometry, image recognition, theoretical physics, signal processing). He publish many paper in different conferences and scientific and scientific journals.

${ }^{2}$ Ali MakkiSagheer was born in basrah - 1961. He got on B.Sc of information system in computer science Department at the university of Technology 2001- Iraq, M.Sc. in data security from the university Technology(2004) and Ph.D. in computer science from the university Technology(2007). He interesting in the follow filed (cryptology, information security, number theory, multimedia compression, image processing, coding system and artificial intelligence). He publish many paper in different conferences and scientific and scientific journals.

${ }^{3}$ Dheyaa Ahmed Ibrahim was born in Anbar - 1988. has received B.Sc in Computer Science, Al-Anbar University, Iraq, (2005-2009) and M.Sc in Computer Science Department, Al-nabar University, (2011). Fields of interest: computer vision, image processing, face recognition, face detection and related fields. He taught many subjects such as operation system, cryptography and encryption.
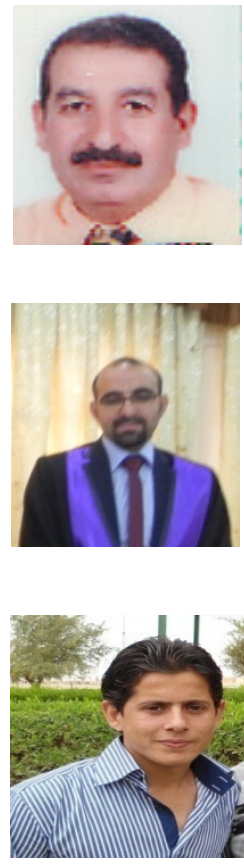